University of Texas at El Paso

ScholarWorks@UTEP

$12-2009$

\title{
Optimal Sensor Placement in Environmental Research: Designing a Sensor Network under Uncertainty
}

\author{
Aline James \\ Craig Tweedie \\ The University of Texas at El Paso, ctweedie@utep.edu \\ Tanja Magoc \\ The University of Texas at El Paso, tmagoc@utep.edu \\ Vladik Kreinovich \\ The University of Texas at El Paso, vladik@utep.edu \\ Martine Ceberio \\ The University of Texas at El Paso, mceberio@utep.edu \\ Follow this and additional works at: https://scholarworks.utep.edu/cs_techrep \\ Part of the Computer Engineering Commons \\ Comments: \\ Technical Report: UTEP-CS-09-30a \\ Published in: Michael Beer, Rafi L. Muhanna, and Robert L. Mullen (Eds.), Proceedings of the 4th \\ International Workshop on Reliable Engineering Computing REC'2010, Singapore, March 3-5, \\ 2010, pp. 255-267.
}

\section{Recommended Citation}

James, Aline; Tweedie, Craig; Magoc, Tanja; Kreinovich, Vladik; and Ceberio, Martine, "Optimal Sensor Placement in Environmental Research: Designing a Sensor Network under Uncertainty" (2009). Departmental Technical Reports (CS). 58.

https://scholarworks.utep.edu/cs_techrep/58

This Article is brought to you for free and open access by the Computer Science at ScholarWorks@UTEP. It has been accepted for inclusion in Departmental Technical Reports (CS) by an authorized administrator of ScholarWorks@UTEP. For more information, please contact Iweber@utep.edu. 


\title{
Optimal Sensor Placement in Environmental Research: Designing a Sensor Network under Uncertainty
}

\author{
Aline Jaimes, Craig Tweedie, Tanja Magoč \\ Vladik Kreinovich, and Martine Ceberio \\ Cyber-ShARE Center \\ University of Texas at El Paso, El Paso, TX 79968, USA \\ contactemailvvladik@utep.edu
}

\begin{abstract}
One of our main challenges in meteorology and environment research is that in many important remote areas, sensor coverage is sparse, leaving us with numerous blind spots. Placement and maintenance of sensors in these areas are expensive. It is therefore desirable to find out how, within a given budget, we can design a sensor network are important activities was developing reasonable techniques for sensor that would provide us with the largest amount of useful information while minimizing the size of the "blind spot" areas which is not covered by the sensors.

This problem is very difficult even to formulate in precise terms because of the huge uncertainty. There are two important aspects of this problem: (1) how to best distribute the sensors over the large area, and (2) what is the best location of each sensor in the corresponding zone. There is some researcj on the first aspect of the problem.

In this paper, we illustrate the second aspect of the problem, on the example of optimal selection of locations for the Eddy towers, an important micrometeorological instrument.
\end{abstract}

Keywords: sensor placement, uncertainty, environmental research

\section{Formulation of the Problem}

Additional sensors are needed. One of our main challenges in meteorology and environment research is that in many important remote areas, sensor coverage is sparse, leaving us with numerous blind spots; see, e.g., (Kintisch, 2009). Placement and maintenance of sensors in these areas are expensive. It is therefore desirable to find out how, within a given budget, we can design a sensor network are important activities was developing reasonable techniques for sensor that would provide us with the largest amount of useful information while minimizing the size of the "blind spot" areas which is not covered by the sensors.

Uncertainty. This problem is very difficult even to formulate in precise terms because of the huge uncertainty.

Two aspects of the problem. There are two important aspects of this problem:

(C) 2010 by authors. Printed in SINGAPORE.

REC 2010 - A. Jaimes, C. Tweedie, T. Magoč, V. Kreinovich, and M. Ceberio 
(1) how to best distribute the sensors over the large area, and

(2) what is the best location of each sensor in the corresponding zone.

There are known methods of dealing with the first aspect of this problem; see, e.g., (Nguyen et al., 2008) and references therein.

Case study. We illustrate how to deal with the second related of the problem on the example of optimal selection of locations for the Eddy towers, an important micrometeorological instrument.

In this selection, we have several criteria to satisfy (Jaimes, 2008).

- For example, the station should not be located too close to a road, so that the gas flux generated by the cars do not influence our measurements of atmospheric fluxes; in other words, the distance $x_{1}$ to the road should be larger than a certain threshold $t_{1}: x_{1}>t_{1}$, or $y_{1} \stackrel{\text { def }}{=} x_{1}-t_{1}>0$.

- Also, the inclination $x_{2}$ at the should be smaller than a corresponding threshold $t_{2}$, because otherwise, the flux will be mostly determined by this inclination and will not be reflective of the atmospheric processes: $x_{2}<t_{2}$, or $y_{2} \stackrel{\text { def }}{=} t_{2}-x_{2}>0$.

General case. In general, we have several such differences $y_{1}, \ldots, y_{n}$ all of which have to be nonnegative. For each of the differences $y_{i}$, the larger its value, the better.

\section{Weighted Average: A Natural Idea and Its Limitations}

Problem: reminder. We want to select the best location based on the values of the differences $y_{1}, \ldots, y_{n}$. For each of the differences $y_{i}$, the larger its value, the better.

Multi-criteria optimization. Our problem is a typical setting for multi-criteria optimization; see, e.g., (Ehrgott and X. Gandibleux, 2002; Sawaragi et al., 1985; Steuer, 1986).

Weighted average. A most widely used approach to multi-criteria optimization is weighted average, where we assign weights $w_{1}, \ldots, w_{n}>0$ to different criteria $y_{i}$ and select an alternative for which the weighted average $w_{1} \cdot y_{1}+\ldots+w_{n} \cdot y_{n}$ attains the largest possible value.

This approach has been used in many practical problems ranging from selecting the lunar landing sites for the Apollo missions (Binder and Roberts, 1970) to selecting landfill sites (Fountoulis et al., 2003).

Additional requirement. In our problem, we have an additional requirement - that all the values $y_{i}$ must be positive. Thus, we must only compare solutions with $y_{i}>0$ when selecting an alternative with the largest possible value of the weighted average. 
Limitations of the weighted average approach. In general, the weighted average approach often leads to reasonable solutions of the multi-criteria optimization problem. However, as we will show, in the presence of the additional positivity requirement, the weighted average approach is not fully satisfactory.

A practical multi-criteria optimization must take into account that measurements are not absolutely accurate. Indeed, the values $y_{i}$ come from measurements, and measurements are never absolutely accurate. The results $\widetilde{y}_{i}$ of the measurements are close to the actual (unknown) values $y_{i}$ of the measured quantities, but they are not exactly equal to these values. If

- we measure the values $y_{i}$ with higher and higher accuracy and,

- based on the measurement results $\widetilde{y}_{i}$, we conclude that the alternative $y=\left(y_{1}, \ldots, y_{n}\right)$ is better than some other alternative $y^{\prime}=\left(y_{1}^{\prime}, \ldots, y_{n}^{\prime}\right)$,

then we expect that the actual alternative $y$ is indeed either better than $y^{\prime}$ or at least of the same quality as $y^{\prime}$. Otherwise, if we do not make this assumption, we will not be able to make any meaningful conclusions based on real-life (approximate) measurements.

The above natural requirement is not always satisfied for weighted average. Let us show that for the weighted average, this "continuity" requirement is not satisfied even in the simplest case when we have only two criteria $y_{1}$ and $y_{2}$. Indeed, let $w_{1}>0$ and $w_{2}>0$ be the weights corresponding to these two criteria. Then, the resulting strict preference relation $\succ$ has the following properties:

- if $y_{1}>0, y_{2}>0, y_{1}^{\prime}>0$, and $y_{2}^{\prime}>0$, and $w_{1} \cdot y_{1}^{\prime}+w_{2} \cdot y_{2}^{\prime}>w_{1} \cdot y_{1}+w_{2} \cdot y_{2}$, then

$$
y^{\prime}=\left(y_{1}^{\prime}, y_{2}^{\prime}\right) \succ y=\left(y_{1}, y_{2}\right) ;
$$

- if $y_{1}>0, y_{2}>0$, and at least one of the values $y_{1}^{\prime}$ and $y_{2}^{\prime}$ is non-positive, then

$$
y=\left(y_{1}, y_{2}\right) \succ y^{\prime}=\left(y_{1}^{\prime}, y_{2}^{\prime}\right) .
$$

Let us consider, for every $\varepsilon>0$, the tuple $y^{\prime}(\varepsilon) \stackrel{\text { def }}{=}\left(\varepsilon, 1+\frac{w_{1}}{w_{2}}\right)$, with $y_{1}^{\prime}(\varepsilon)=\varepsilon$ and $y_{2}^{\prime}(\varepsilon)=1+\frac{w_{1}}{w_{2}}$, and also the comparison tuple $y=(1,1)$. In this case, for every $\varepsilon>0$, we have

$$
w_{1} \cdot y_{1}^{\prime}(\varepsilon)+w_{2} \cdot y_{2}^{\prime}(\varepsilon)=w_{1} \cdot \varepsilon+w_{2}+w_{2} \cdot \frac{w_{1}}{w_{2}}=w_{1} \cdot(1+\varepsilon)+w_{2}
$$

and

$$
w_{1} \cdot y_{1}+w_{2} \cdot y_{2}=w_{1}+w_{2},
$$

hence $y^{\prime}(\varepsilon) \succ y$. However, in the limit $\varepsilon \rightarrow 0$, we have $y^{\prime}(0)=\left(0,1+\frac{w_{1}}{w_{2}}\right)$, with $y_{1}^{\prime}(0)=0$ and thus, $y^{\prime}(0) \prec y$. 


\section{Towards a More Adequate Approach to Multi-Criterion Optimization}

What we want: a precise description. We want to be able to compare different alternatives.

Each alternative is characterized by a tuple of $n$ values $y=\left(y_{1}, \ldots, y_{n}\right)$, and only alternatives for which all the values $y_{i}$ are positive are allowed. Thus, from the mathematical viewpoint, the set of all alternatives is the set $\left(R^{+}\right)^{n}$ of all the tuples of positive numbers.

For each two alternatives $y$ and $y^{\prime}$, we want to tell whether $y$ is better than $y^{\prime}$ (we will denote it by $y \succ y^{\prime}$ or $\left.y^{\prime} \prec y\right)$, or $y^{\prime}$ is better than $y\left(y^{\prime} \succ y\right)$, or $y$ and $y^{\prime}$ are equally good $\left(y^{\prime} \sim y\right)$. These relations must satisfy natural properties. For example, if $y$ is better than $y^{\prime}$ and $y^{\prime}$ is better than $y^{\prime \prime}$, then $y$ is better than $y^{\prime \prime}$. In other words, the relation $\succ$ must be transitive. Similarly, the relation $\sim$ must be transitive, symmetric, and reflexive $(y \sim y)$, i.e., in mathematical terms, an equivalence relation.

So, we want to define a pair of relations $\succ$ and $\sim$ such that $\succ$ is transitive, $\sim$ is transitive, $\sim$ is an equivalence relation, and for every $y$ and $y^{\prime}$, one and only one of the following relations hold: $y \succ y^{\prime}, y^{\prime} \succ y$, or $y \sim y^{\prime}$.

It is also reasonable to require that if each criterion is better, then the alternative is better as well, i.e., that if $y_{i}>y_{i}^{\prime}$ for all $i$, then $y \succ y^{\prime}$.

Comment. Pairs of relations of the above type can be alternatively characterized by a pre-ordering relation

$$
y^{\prime} \succeq y \Leftrightarrow\left(y^{\prime} \succ y \vee y^{\prime} \sim y\right)
$$

This relation must be transitive and - in our case - total (i.e., for every $y$ and $y^{\prime}$, we have $y \succeq$ $y^{\prime} \vee y^{\prime} \succeq y$. Once we know the pre-ordering relation $\succeq$, we can reconstruct $\succ$ and $\sim$ as follows:

$$
\begin{aligned}
& y^{\prime} \succ y \Leftrightarrow\left(y^{\prime} \succeq y \& y \succeq y^{\prime}\right) ; \\
& y^{\prime} \sim y \Leftrightarrow\left(y^{\prime} \succeq y \& y \succeq y^{\prime}\right) .
\end{aligned}
$$

Scale invariance: motivation. The quantities $y_{i}$ describe completely different physical notions, measured in completely different units. In our meteorological case, some of these values are wind velocities measured in meters per second, or in kilometers per hour, or miles per hour. Other values are elevations described in meters, kilometers, or feet, etc. Each of these quantities can be described in many different units. A priori, we do not know which units match each other, so it is reasonable to assume that the units used for measuring different quantities may not be exactly matched.

It is therefore reasonable to require that the relations $\succ$ and $\sim$ between the two alternatives $y=\left(y_{1}, \ldots, y_{n}\right)$ and $y^{\prime}=\left(y_{1}^{\prime}, \ldots, y_{n}^{\prime}\right)$ do not change if we simply change the units in which we measure each of the corresponding $n$ quantities.

Comment. The importance of such invariance is well known in measurements theory, starting with the pioneering work on S. S. Stevens on (Stevens, 1964); see also the classical books (Pfanzangl, 1968) and (Luce et al., 1990) (especially Chapter 22), where this invariance is also called meaningfulness. 
Scale invariance: towards a precise description. When we replace a unit in which we measure a certain quantity $q$ by a new measuring unit which is $\lambda>0$ times smaller, then the numerical values of this quantity increase by a factor of $\lambda: q \rightarrow \lambda \cdot q$. For example, $1 \mathrm{~cm}$ is $\lambda=100$ times smaller than $1 \mathrm{~m}$, so the length $q=2 \mathrm{~m}$, when measured in $\mathrm{cm}$, becomes $\lambda \cdot q=2 \cdot 100=200 \mathrm{~cm}$.

Let $\lambda_{i}$ denote the ratio of the old to the new units corresponding to the $i$-th quantity. Then, the quantity that had the value $y_{i}$ in the old units will be described by a numerical value $\lambda_{i} \cdot y_{i}$ in the new units. Therefore, scale-invariance means that for all $y, y^{\prime} \in\left(R^{+}\right)^{n}$ and for all $\lambda_{i}>0$, we have

$$
y^{\prime}=\left(y_{1}^{\prime}, \ldots, y_{n}^{\prime}\right) \succ y=\left(y_{1}, \ldots, y_{n}\right) \Rightarrow\left(\lambda_{1} \cdot y_{1}^{\prime}, \ldots, \lambda_{n} \cdot y_{n}^{\prime}\right) \succ\left(\lambda_{1} \cdot y_{1}, \ldots, \lambda_{n} \cdot y_{n}\right)
$$

and

$$
y^{\prime}=\left(y_{1}^{\prime}, \ldots, y_{n}^{\prime}\right) \sim y=\left(y_{1}, \ldots, y_{n}\right) \Rightarrow\left(\lambda_{1} \cdot y_{1}^{\prime}, \ldots, \lambda_{n} \cdot y_{n}^{\prime}\right) \sim\left(\lambda_{1} \cdot y_{1}, \ldots, \lambda_{n} \cdot y_{n}\right)
$$

Comment. In general, in measurements, in addition to changing the unit, we can also change the starting point. However, for the differences $y_{i}$, the starting point is fixed by the fact that 0 corresponds to the threshold value. So, in our case, only changing a measuring unit (= scaling) makes sense.

Continuity. As we have mentioned in the previous section, we also want to require that the relations $\succ$ and $\sim$ are continuous in the following sense: if $y^{\prime}(\varepsilon) \succeq y(\varepsilon)$ for every $\varepsilon$, then in the limit, when $y^{\prime}(\varepsilon) \rightarrow y^{\prime}(0)$ and $y(\varepsilon) \rightarrow y(0)$ (in the sense of normal convergence in $R^{n}$ ), we should have $y^{\prime}(0) \succeq y(0)$.

Let us now describe our requirements in precise terms.

Definition 1. By a total pre-ordering relation on a set $Y$, we mean a pair of a transitive relation $\succ$ and an equivalence relation $\sim$ for which, for every $y, y^{\prime} \in Y$, one and only one of the following relations hold: $y \succ y^{\prime}, y^{\prime} \succ y$, or $y \sim y^{\prime}$.

Comment. We will denote $y \succeq y^{\prime} \stackrel{\text { def }}{=}\left(y \succ y^{\prime} \vee y \sim y^{\prime}\right)$.

Definition 2. We say that a total pre-ordering is non-trivial if there exist $y$ and $y^{\prime}$ for which $y^{\prime} \succ y$.

Comment. This definition excludes the trivial pre-ordering in which every two tuples are equivalent to each other. 
Definition 3. We say that a total pre-ordering relation on the set $\left(R^{+}\right)^{n}$ is:

- monotonic if $y_{i}^{\prime}>y_{i}$ for all $i$ implies $y^{\prime} \succ y$;

- scale-invariant if for all $\lambda_{i}>0$ :

- $\left(y_{1}^{\prime}, \ldots, y_{n}^{\prime}\right) \succ y=\left(y_{1}, \ldots, y_{n}\right)$ implies $\left(\lambda_{1} \cdot y_{1}^{\prime}, \ldots, \lambda_{n} \cdot y_{n}^{\prime}\right) \succ\left(\lambda_{1} \cdot y_{1}, \ldots, \lambda_{n} \cdot y_{n}\right)$, and

- $\left(y_{1}^{\prime}, \ldots, y_{n}^{\prime}\right) \sim y=\left(y_{1}, \ldots, y_{n}\right)$ implies $\left(\lambda_{1} \cdot y_{1}^{\prime}, \ldots, \lambda_{n} \cdot y_{n}^{\prime}\right) \sim\left(\lambda_{1} \cdot y_{1}, \ldots, \lambda_{n} \cdot y_{n}\right)$.

- continuous if whenever we have a sequence $y^{(k)}$ of tuples for which $y^{(k)} \succeq y^{\prime}$ for some tuple $y^{\prime}$, and the sequence $y^{(k)}$ tends to a limit $y$, then $y \succeq y^{\prime}$.

Theorem. Every non-trivial monotonic scale-invariant continuous total pre-ordering relation on $\left(R^{+}\right)^{n}$ has the following form:

$$
\begin{aligned}
& y^{\prime}=\left(y_{1}^{\prime}, \ldots, y_{n}^{\prime}\right) \succ y=\left(y_{1}, \ldots, y_{n}\right) \Leftrightarrow \prod_{i=1}^{n}\left(y_{i}^{\prime}\right)^{\alpha_{i}}>\prod_{i=1}^{n} y_{i}^{\alpha_{i}} ; \\
& y^{\prime}=\left(y_{1}^{\prime}, \ldots, y_{n}^{\prime}\right) \sim y=\left(y_{1}, \ldots, y_{n}\right) \Leftrightarrow \prod_{i=1}^{n}\left(y_{i}^{\prime}\right)^{\alpha_{i}}=\prod_{i=1}^{n} y_{i}^{\alpha_{i}},
\end{aligned}
$$

for some constants $\alpha_{i}>0$.

Comment. In other words, for every non-trivial monotonic scale-invariant continuous total preordering relation on $\left(R^{+}\right)^{n}$, there exist values $\alpha_{1}>0, \ldots, \alpha_{n}>0$ for which the above equivalence hold. Vice versa, for each set of values $\alpha_{1}>0, \ldots, \alpha_{n}>0$, the above formulas define a monotonic scale-invariant continuous pre-ordering relation on $\left(R^{+}\right)^{n}$.

It is worth mentioning that the resulting relation coincides with the asymmetric version (Roth, 1979) of the bargaining solution proposed by the Nobelist John Nash in 1953 (Nash, 2008).

\section{Proof}

$1^{\circ}$. Due to scale-invariance, for every $y_{1}, \ldots, y_{n}, y_{1}^{\prime}, \ldots, y_{n}^{\prime}$, we can take $\lambda_{i}=\frac{1}{y_{i}}$ and conclude that

$$
\left(y_{1}^{\prime}, \ldots, y_{n}^{\prime}\right) \sim\left(y_{1}, \ldots, y_{n}\right) \Leftrightarrow\left(\frac{y_{1}^{\prime}}{y_{1}}, \ldots, \frac{y_{n}^{\prime}}{y_{n}}\right) \sim(1, \ldots, 1) .
$$

Thus, to describe the equivalence relation $\sim$, it is sufficient to describe the set of all the vectors $z=\left(z_{1}, \ldots, z_{n}\right)$ for which $z \sim(1, \ldots, 1)$. Similarly,

$$
\left(y_{1}^{\prime}, \ldots, y_{n}^{\prime}\right) \succ\left(y_{1}, \ldots, y_{n}\right) \Leftrightarrow\left(\frac{y_{1}^{\prime}}{y_{1}}, \ldots, \frac{y_{n}^{\prime}}{y_{n}}\right) \succ(1, \ldots, 1) .
$$


Thus, to describe the ordering relation $\succ$, it is sufficient to describe the set of all the vectors $z=\left(z_{1}, \ldots, z_{n}\right)$ for which $z \succ(1, \ldots, 1)$.

Alternatively, we can take $\lambda_{i}=\frac{1}{y_{i}^{\prime}}$ and conclude that

$$
\left(y_{1}^{\prime}, \ldots, y_{n}^{\prime}\right) \succ\left(y_{1}, \ldots, y_{n}\right) \Leftrightarrow(1, \ldots, 1) \succ\left(\frac{y_{1}}{y_{1}^{\prime}}, \ldots, \frac{y_{n}}{y_{n}^{\prime}}\right) .
$$

Thus, it is also sufficient to describe the set of all the vectors $z=\left(z_{1}, \ldots, z_{n}\right)$ for which $(1, \ldots, 1) \succ z$.

$2^{\circ}$. The above equivalence involves division. To simplify the description, we can take into account that in the logarithmic space, division becomes a simple difference: $\ln \left(\frac{y_{i}^{\prime}}{y_{i}}\right)=\ln \left(y_{i}^{\prime}\right)-\ln \left(y_{i}\right)$. To use this simplification, let us consider the logarithms $Y_{i} \stackrel{\text { def }}{=} \ln \left(y_{i}\right)$ of different values. In terms of these logarithms, the original values can be reconstructed as $y_{i}=\exp \left(Y_{i}\right)$. In terms of these logarithms, we thus need to consider:

- the set $S_{\sim}$ of all the tuples $Z=\left(Z_{1}, \ldots, Z_{n}\right)$ for which $z=\left(\exp \left(Z_{1}\right), \ldots, \exp \left(Z_{n}\right)\right) \sim(1, \ldots, 1)$, and

- the set $S_{\succ}$ of all the tuples $Z=\left(Z_{1}, \ldots, Z_{n}\right)$ for which $z=\left(\exp \left(Z_{1}\right), \ldots, \exp \left(Z_{n}\right)\right) \succ(1, \ldots, 1)$.

We will also consider the set $S_{\prec}$ of all the tuples $Z=\left(Z_{1}, \ldots, Z_{n}\right)$ for which

$$
(1, \ldots, 1) \succ z=\left(\exp \left(Z_{1}\right), \ldots, \exp \left(Z_{n}\right)\right) .
$$

Since the pre-ordering relation is total, for every tuple $z$,

- either $z \sim(1, \ldots, 1)$,

- or $z \succ(1, \ldots, 1)$,

- or $(1, \ldots, 1) \succ z$.

In particular, this is true for $z=\left(\exp \left(Z_{1}\right), \ldots, \exp \left(Z_{n}\right)\right)$. Thus, for every tuple $Z$, either $Z \in S_{\sim}$ or $Z \in S_{\succ}$ or $Z \in S_{\prec}$.

$3^{\circ}$. Let us prove that the set $S_{\sim}$ is closed under addition, i.e., that if the tuples $Z=\left(Z_{1}, \ldots, Z_{n}\right)$ and $Z^{\prime}=\left(Z_{1}^{\prime}, \ldots, Z_{n}^{\prime}\right)$ belong to the set $S_{\sim}$, then their component-wise sum

$$
Z+Z^{\prime}=\left(Z_{1}+Z_{1}^{\prime}, \ldots, Z_{n}+Z_{n}^{\prime}\right)
$$

also belongs to the set $S_{\sim}$.

Indeed, by definition of the set $S_{\sim}$, the condition $Z \in S_{\sim}$ means that

$$
\left(\exp \left(Z_{1}\right), \ldots, \exp \left(Z_{n}\right)\right) \sim(1, \ldots, 1) .
$$


Using scale-invariance with $\lambda_{i}=\exp \left(Z_{i}^{\prime}\right)$, we conclude that

$$
\left(\exp \left(Z_{1}\right) \cdot \exp \left(Z_{1}^{\prime}\right), \ldots, \exp \left(Z_{n}\right) \cdot \exp \left(Z_{n}^{\prime}\right)\right) \sim\left(\exp \left(Z_{1}^{\prime}\right), \ldots, \exp \left(Z_{n}^{\prime}\right)\right) .
$$

On the other hand, the condition $Z^{\prime} \in S_{\sim}$ means that

$$
\left(\exp \left(Z_{1}^{\prime}\right), \ldots, \exp \left(Z_{n}^{\prime}\right)\right) \sim(1, \ldots, 1) .
$$

Thus, due to transitivity of the equivalence relation $\sim$, we conclude that

$$
\left(\exp \left(Z_{1}\right) \cdot \exp \left(Z_{1}^{\prime}\right), \ldots, \exp \left(Z_{n}\right) \cdot \exp \left(Z_{n}^{\prime}\right)\right) \sim(1, \ldots, 1) .
$$

Since for every $i$, we have $\exp \left(Z_{i}\right) \cdot \exp \left(Z_{i}^{\prime}\right)=\exp \left(Z_{i}+Z_{i}^{\prime}\right)$, we thus conclude that

$$
\left(\exp \left(Z_{1}+Z_{1}^{\prime}\right), \ldots, \exp \left(Z_{n}+Z_{n}^{\prime}\right)\right) \sim(1, \ldots, 1) .
$$

By definition of the set $S_{\sim}$, this means that the tuple $Z+Z^{\prime}$ belongs to the set $S_{\sim}$.

$4^{\circ}$. Similarly, we can prove that the set $S_{\succ}$ is closed under addition, i.e., that if the tuples $Z=$ $\left(Z_{1}, \ldots, Z_{n}\right)$ and $Z^{\prime}=\left(Z_{1}^{\prime}, \ldots, Z_{n}^{\prime}\right)$ belong to the set $S_{\succ}$, then their component-wise sum

$$
Z+Z^{\prime}=\left(Z_{1}+Z_{1}^{\prime}, \ldots, Z_{n}+Z_{n}^{\prime}\right)
$$

also belongs to the set $S_{\succ}$.

Indeed, by definition of the set $S_{\succ}$, the condition $Z \in S_{\succ}$ means that

$$
\left(\exp \left(Z_{1}\right), \ldots, \exp \left(Z_{n}\right)\right) \succ(1, \ldots, 1) .
$$

Using scale-invariance with $\lambda_{i}=\exp \left(Z_{i}^{\prime}\right)$, we conclude that

$$
\left(\exp \left(Z_{1}\right) \cdot \exp \left(Z_{1}^{\prime}\right), \ldots, \exp \left(Z_{n}\right) \cdot \exp \left(Z_{n}^{\prime}\right)\right) \succ\left(\exp \left(Z_{1}^{\prime}\right), \ldots, \exp \left(Z_{n}^{\prime}\right)\right) .
$$

On the other hand, the condition $Z^{\prime} \in S_{\succ}$ means that

$$
\left(\exp \left(Z_{1}^{\prime}\right), \ldots, \exp \left(Z_{n}^{\prime}\right)\right) \succ(1, \ldots, 1) .
$$

Thus, due to transitivity of the strict preference relation $\succ$, we conclude that

$$
\left(\exp \left(Z_{1}\right) \cdot \exp \left(Z_{1}^{\prime}\right), \ldots, \exp \left(Z_{n}\right) \cdot \exp \left(Z_{n}^{\prime}\right)\right) \succ(1, \ldots, 1) .
$$

Since for every $i$, we have $\exp \left(Z_{i}\right) \cdot \exp \left(Z_{i}^{\prime}\right)=\exp \left(Z_{i}+Z_{i}^{\prime}\right)$, we thus conclude that

$$
\left(\exp \left(Z_{1}+Z_{1}^{\prime}\right), \ldots, \exp \left(Z_{n}+Z_{n}^{\prime}\right)\right) \succ(1, \ldots, 1) .
$$

By definition of the set $S_{\succ}$, this means that the tuple $Z+Z^{\prime}$ belongs to the set $S_{\succ}$.

$5^{\circ}$. A similar argument shows that the set $S_{\prec}$ is closed under addition, i.e., that if the tuples $Z=\left(Z_{1}, \ldots, Z_{n}\right)$ and $Z^{\prime}=\left(Z_{1}^{\prime}, \ldots, Z_{n}^{\prime}\right)$ belong to the set $S_{\prec}$, then their component-wise sum

$$
Z+Z^{\prime}=\left(Z_{1}+Z_{1}^{\prime}, \ldots, Z_{n}+Z_{n}^{\prime}\right)
$$


also belongs to the set $S_{\prec}$.

$6^{\circ}$. Let us now prove that the set $S_{\sim}$ is closed under the "unary minus" operation, i.e., that if $Z=\left(Z_{1}, \ldots, Z_{n}\right) \in S_{\sim}$, then $-Z \stackrel{\text { def }}{=}\left(-Z_{1}, \ldots,-Z_{n}\right)$ also belongs to $S_{\sim}$.

Indeed, $Z \in S_{\sim}$ means that

$$
\left(\exp \left(Z_{1}\right), \ldots, \exp \left(Z_{n}\right)\right) \sim(1, \ldots, 1) .
$$

Using scale-invariance with $\lambda_{i}=\exp \left(-Z_{i}\right)=\frac{1}{\exp \left(Z_{i}\right)}$, we conclude that

$$
(1, \ldots, 1) \sim\left(\exp \left(-Z_{1}\right), \ldots, \exp \left(-Z_{n}\right)\right)
$$

i.e., that $-Z \in S_{\sim}$.

$7^{\circ}$. Let us prove that if $Z=\left(Z_{1}, \ldots, Z_{n}\right) \in S_{\succ}$, then $-Z \stackrel{\text { def }}{=}\left(-Z_{1}, \ldots,-Z_{n}\right)$ belongs to $S_{\prec}$.

Indeed, $Z \in S_{\succ}$ means that

$$
\left(\exp \left(Z_{1}\right), \ldots, \exp \left(Z_{n}\right)\right) \succ(1, \ldots, 1) .
$$

Using scale-invariance with $\lambda_{i}=\exp \left(-Z_{i}\right)=\frac{1}{\exp \left(Z_{i}\right)}$, we conclude that

$$
(1, \ldots, 1) \succ\left(\exp \left(-Z_{1}\right), \ldots, \exp \left(-Z_{n}\right)\right),
$$

i.e., that $-Z \in S_{\prec}$.

Similarly, we can show that if $Z \in S_{\prec}$, then $-Z \in S_{\succ}$.

$8^{\circ}$. From Part 3 of this proof, it now follows that if $Z=\left(Z_{1}, \ldots, Z_{n}\right) \in S_{\sim}$, then $Z+Z \in S_{\sim}$, that $Z+(Z+Z) \in S_{\sim}$, etc., i.e., that for every positive integer $p$, the tuple

$$
p \cdot Z=\left(p \cdot Z_{1}, \ldots, p \cdot Z_{n}\right)
$$

also belongs to the set $S_{\sim}$.

By using Part 6, we can also conclude that this is true for negative integers $p$ as well. Finally, by taking into account that the zero tuple $0 \stackrel{\text { def }}{=}(0, \ldots, 0)$ can be represented as $Z+(-Z)$, we conclude that $0 \cdot Z=0$ also belongs to the set $S_{\sim}$.

Thus, if a tuple $Z$ belongs to the set $S_{\sim}$, then for every integer $p$, the tuple $p \cdot Z$ also belongs to the set $S_{\sim}$.

$9^{\circ}$. Similarly, from Parts 4 and 5 of this proof, it follows that

- if $Z=\left(Z_{1}, \ldots, Z_{n}\right) \in S_{\succ}$, then for every positive integer $p$, the tuple $p \cdot Z$ also belongs to the set $S_{\succ}$, and

- if $Z=\left(Z_{1}, \ldots, Z_{n}\right) \in S_{\prec}$, then for every positive integer $p$, the tuple $p \cdot Z$ also belongs to the set $S_{\prec}$. 
$10^{\circ}$. Let us prove that for every rational number $r=\frac{p}{q}$, where $p$ is an integer and $q$ is a positive integer, if a tuple $Z$ belongs to the set $S_{\sim}$, then the tuple $r \cdot Z$ also belongs to the set $S_{\sim}$.

Indeed, according to Part $8, Z \in S_{\sim}$ implies that $p \cdot Z \in S_{\sim}$.

According to Part 2, for the tuple $r \cdot Z$, we have either $r \cdot Z \in S_{\sim}$, or $r \cdot Z \in S_{\succ}$, or $r \cdot Z \in S_{\prec}$.

- If $r \cdot Z \in S_{\succ}$, then, by Part 9 , we would get $p \cdot Z=q \cdot(r \cdot Z) \in S_{\succ}$, which contradicts our result that $p \cdot Z \in S_{\sim}$.

- Similarly, if $r \cdot Z \in S_{\prec}$, then, by Part 9 , we would get $p \cdot Z=q \cdot(r \cdot Z) \in S_{\prec}$, which contradicts our result that $p \cdot Z \in S_{\sim}$.

Thus, the only remaining option is $r \cdot Z \in S_{\sim}$. The statement is proven.

$11^{\circ}$. Let us now use continuity to prove that for every real number $x$, if a tuple $Z$ belongs to the set $S_{\sim}$, then the tuple $x \cdot Z$ also belongs to the set $S_{\sim}$.

Indeed, a real number $x$ can be represented as a limit of rational numbers: $r^{(k)} \rightarrow x$. According to Part 10 , for every $k$, we have $r^{(k)} \cdot Z \in S_{\sim}$, i.e., the tuple

$$
Z^{(k)} \stackrel{\text { def }}{=}\left(\exp \left(r^{(k)} \cdot Z_{1}\right), \ldots, \exp \left(r^{(k)} \cdot Z_{n}\right)\right) \sim(1, \ldots, 1) .
$$

In particular, this means that $Z^{(k)} \succeq(1, \ldots, 1)$. In the limit,

$$
Z^{(k)} \rightarrow\left(\exp \left(x \cdot Z_{1}\right), \ldots, \exp \left(x \cdot Z_{n}\right)\right) \succeq(1, \ldots, 1) .
$$

By definition of the sets $S_{\sim}$ and $S_{\succ}$, this means that $x \cdot Z \in S_{\sim}$ or $x \cdot Z \in S_{\succ}$.

Similarly, for $-(x \cdot Z)=(-x) \cdot Z$, we conclude that $-x \cdot Z \in S_{\sim}$ or $(-x) \cdot Z \in S_{\succ}$. If we had $x \cdot Z \in S_{\succ}$, then by Part 7 we would get $(-x) \cdot Z \in S_{\prec}$, a contradiction. Thus, the case $x \cdot Z \in S_{\succ}$ is impossible, and we have $x \cdot Z \in S_{\sim}$. The statement is proven.

$12^{\circ}$. According to Parts 3 and 11, the set $S_{\sim}$ is closed under addition and under multiplication by an arbitrary real number. Thus, if tuples $Z, \ldots, Z^{\prime}$ belong to the set $S_{\sim}$, their arbitrary linear combination $x \cdot Z+\ldots+x^{\prime} \cdot Z^{\prime}$ also belongs to the set $S_{\sim}$. So, the set $S_{\sim}$ is a linear subspace of the $n$-dimensional space of all the tuples.

$13^{\circ}$. The subspace $S_{\sim}$ cannot coincide with the entire $n$-dimensional space, because then the preordering relation would be trivial. Thus, the dimension of this subspace must be less than or equal to $n-1$. Let us show that the dimension of this subspace is $n-1$.

Indeed, let us assume that the dimension is smaller than $n-1$. Since the pre-ordering is nontrivial, there exist tuples $y=\left(y_{1}, \ldots, y_{n}\right)$ and $y^{\prime}=\left(y_{1}^{\prime}, \ldots, y_{n}^{\prime}\right)$ for which $y \succ y^{\prime}$ and thus, $Z=$ $\left(Z_{1}, \ldots, Z_{n}\right) \in S_{\succ}$, where $Z_{i}=\ln \left(\frac{y_{i}}{y_{i}^{\prime}}\right)$. From $Z \in S_{\succ}$, we conclude that $-Z \in S_{\prec}$.

Since the linear space $S_{\sim}$ is a less than $(n-1)$-dimensional subspace of an $n$-dimensional linear space, there is a path connecting $Z \in S_{\succ}$ and $-Z \in S_{\prec}$ which avoids $S_{\sim}$. In mathematical terms, this path is a continuous mapping $\gamma:[0,1] \rightarrow R^{n}$ for which $\gamma(0)=Z$ and $\gamma(1)=-Z$. Since this path avoids $S_{\sim}$, every point $\gamma(t)$ on this path belongs either to $S_{\succ}$ or to $S_{\prec}$. 
Let $\bar{t}$ denote the supremum (least upper bound) of the set of all the values $t$ for which $\gamma(t) \in S_{\succ}$. By definition of the supremum, there exists a sequence $t^{(k)} \rightarrow \bar{t}$ for which $\gamma\left(t^{(k)}\right) \in S_{\succ}$. Similarly to Part 11, we can use continuity to prove that in the limit, $\gamma(\bar{t}) \in S_{\succ}$ or $\gamma(\bar{t}) \in S_{\sim}$. Since the path avoids the set $S_{\sim}$, we thus get $\gamma(\bar{t}) \in S_{\succ}$.

Similarly, since $\gamma(1) \notin S_{\succ}$, there exists a sequence $t^{(k)} \downarrow \bar{t}$ for which $\gamma\left(t^{(k)}\right) \in S_{\prec}$. We can therefore conclude that in the limit, $\gamma(\bar{t}) \in S_{\succ}$ or $\gamma(\bar{t}) \in S_{\sim}$ - a contradiction with our previous conclusion that $\gamma(\bar{t}) \in S_{\succ}$.

This contradiction shows that the linear space $S_{\sim}$ cannot have dimension $<n-1$ and thus, that this space have dimension $n-1$.

$14^{\circ}$. Every $(n-1)$-dimensional linear subspace of an $n$-dimensional superspace separates the superspace into two half-spaces. Let us show that one of these half-spaces is $S_{\succ}$ and the other is $S_{\prec}$.

Indeed, if one of the subspaces contains two tuples $Z$ and $Z^{\prime}$ for which $Z \in S_{\succ}$ and $Z^{\prime} \in S_{\prec}$, then the line segment $\gamma(t)=t \cdot Z+(1-t) \cdot Z^{\prime}$ containing these two points also belongs to the same subspace, i.e., avoids the set $S_{\sim}$. Thus, similarly to Part 13, we would get a contradiction.

So, if one point from a half-space belongs to $S_{\succ}$, all other points from this subspace also belong to the set $S_{\succ}$. Similarly, if one point from a half-space belongs to $S_{\prec}$, all other points from this subspace also belong to the set $S_{\prec}$.

$15^{\circ}$. Every $(n-1)$-dimensional linear subspace of an $n$-dimensional space has the form

$$
\alpha_{1} \cdot Z_{1}+\ldots+\alpha_{n} \cdot Z_{n}=0
$$

for some real values $\alpha_{i}$, and the corresponding half-spaces have the form

$$
\alpha_{1} \cdot Z_{1}+\ldots+\alpha_{n} \cdot Z_{n}>0
$$

and

$$
\alpha_{1} \cdot Z_{1}+\ldots+\alpha_{n} \cdot Z_{n}<0 .
$$

The set $S_{\succ}$ coincides with one of these subspaces. If it coincides with the set of all tuples $Z$ for which $\alpha_{1} \cdot Z_{1}+\ldots+\alpha_{n} \cdot Z_{n}<0$, then we can rewrite it as $\left(-\alpha_{1}\right) \cdot Z_{1}+\ldots+\left(-\alpha_{n}\right) \cdot Z_{n}>0$, i.e., as $\alpha_{1}^{\prime} \cdot Z_{1}+\ldots+\alpha_{n}^{\prime} \cdot Z_{n}>0$ for $\alpha_{i}^{\prime}=-\alpha_{i}$.

Thus, without losing generality, we can conclude that the set $S_{\succ}$ coincides with the set of all the tuples $Z$ for which $\alpha_{1} \cdot Z_{1}+\ldots+\alpha_{n} \cdot Z_{n}>0$. We have mentioned that

$$
y^{\prime}=\left(y_{1}^{\prime}, \ldots, y_{n}^{\prime}\right) \succ y=\left(y_{1}, \ldots, y_{n}\right) \Leftrightarrow\left(Z_{1}, \ldots, Z_{n}\right) \in S_{\succ},
$$

where $Z_{i}=\ln \left(\frac{y_{i}^{\prime}}{y_{i}}\right)$. Thus,

$$
y^{\prime} \succ y \Leftrightarrow \alpha_{1} \cdot Z_{1}+\ldots+\alpha_{n} \cdot Z_{n}=\alpha_{1} \cdot \ln \left(\frac{y_{1}^{\prime}}{y_{1}}\right)+\ldots+\alpha_{n} \cdot \ln \left(\frac{y_{n}^{\prime}}{y_{n}}\right)>0 .
$$

Since $\ln \left(\frac{y_{i}^{\prime}}{y_{i}}\right)=\ln \left(y_{i}^{\prime}\right)-\ln \left(y_{i}\right)$, the last inequality is equivalent to

$$
\alpha_{1} \cdot \ln \left(y_{1}^{\prime}\right)+\ldots+\alpha_{n} \cdot \ln \left(y_{n}^{\prime}\right)>\alpha_{1} \cdot \ln \left(y_{1}\right)+\ldots+\alpha_{n} \cdot \ln \left(y_{n}\right) .
$$


Let us take exp of both sides; then, due to the monotonicity of the exponential function, we get an equivalent inequality

$$
\exp \left(\alpha_{1} \cdot \ln \left(y_{1}^{\prime}\right)+\ldots+\alpha_{n} \cdot \ln \left(y_{n}^{\prime}\right)\right)>\exp \left(\alpha_{1} \cdot \ln \left(y_{1}\right)+\ldots+\alpha_{n} \cdot \ln \left(y_{n}\right)\right) .
$$

Here,

$$
\exp \left(\alpha_{1} \cdot \ln \left(y_{1}^{\prime}\right)+\ldots+\alpha_{n} \cdot \ln \left(y_{n}^{\prime}\right)\right)=\exp \left(\alpha_{1} \cdot \ln \left(y_{1}^{\prime}\right)\right) \cdot \ldots \cdot \exp \left(\alpha_{n} \cdot \ln \left(y_{n}^{\prime}\right)\right),
$$

where for every $i, e^{\alpha_{i} \cdot z_{i}}=\left(e^{z_{i}}\right)^{\alpha_{i}}$, with $z_{i} \stackrel{\text { def }}{=} \ln \left(y_{i}^{\prime}\right)$, implies that

$$
\exp \left(\alpha_{i} \cdot \ln \left(y_{i}^{\prime}\right)\right)=\left(\exp \left(\ln \left(y_{i}^{\prime}\right)\right)\right)^{\alpha_{i}}=\left(y_{i}^{\prime}\right)^{\alpha_{i}},
$$

so

$$
\exp \left(\alpha_{1} \cdot \ln \left(y_{1}^{\prime}\right)+\ldots+\alpha_{n} \cdot \ln \left(y_{n}^{\prime}\right)\right)=\left(y_{1}^{\prime}\right)^{\alpha_{1}} \cdot \ldots \cdot\left(y_{n}^{\prime}\right)^{\alpha_{n}}
$$

and similarly,

$$
\exp \left(\alpha_{1} \cdot \ln \left(y_{1}\right)+\ldots+\alpha_{n} \cdot \ln \left(y_{n}\right)\right)=y_{1}^{\alpha_{1}} \cdot \ldots \cdot y_{n}^{\alpha_{n}} .
$$

Thus, the condition $y^{\prime} \succ y$ is equivalent

$$
\prod_{i=1}^{n} y_{i}^{\alpha_{i}}>\prod_{i=1}^{n}\left(y_{i}^{\prime}\right)^{\alpha_{i}}
$$

Similarly, we prove that

$$
\left(y_{1}, \ldots, y_{n}\right) \sim y^{\prime}=\left(y_{1}^{\prime}, \ldots, y_{n}^{\prime}\right) \Leftrightarrow \prod_{i=1}^{n} y_{i}^{\alpha_{i}}=\prod_{i=1}^{n}\left(y_{i}^{\prime}\right)^{\alpha_{i}} .
$$

The condition $\alpha_{i}>0$ follows from our assumption that the pre-ordering is monotonic.

The theorem is proven.

Acknowledgments. This work was supported in part by the National Science Foundation grant HRD-0734825.

\section{References}

Binder, A. B. and D. L. Roberts, Criteria for Lunar Site Selection, Report No. P-30, NASA Appollo Lunar Exploration Office and Illinois Institute of Technology Research Institute, Chicago, Illinois, January 1970.

Ehrgott, M. and X. Gandibleux (eds.), Multiple Criteria Optimization: State of the Art Annotated Bibliographic Surveys, Springer Verlag, Berlin-Heidelberg-New York, 2002.

Fountoulis, I., D. Mariokalos, E. Spyridonos, and E. Andreakis, Geological criteria and methodology for landfill sites selection, In: Proceedings of the 8th International Conference on Environmental Science and Technology, Lemnos Island, Greece, September 8-10, 2003, 200-207.

Jaimes, A. A cyber-tool to optimize site selection for establishing an eddy covraince and robotic tram system at the Jornada Experimental Range, University of Texas at El Paso, December 2008.

Kintisch, E. Loss of carbon observatory highlights gaps in data. Science, 323:1276-1277, 2009. 
Luce, R. D., D. H. Krantz, P. Suppes, and A. Tversky, Foundations of Measurement, Vol. 3, Representation, Axiomatization, and Invariance, Academic Press, San Diego, California, 1990.

Nash, J. Two-Person Cooperative Games," Econometrica, 21:128-140, 1953.

Nguyen, H. T., O. Kosheleva, V. Kreinovich, and S. Ferson. Trade-Off Between Sample Size and Accuracy: Cases of Static and Dynamic Measurements under Interval Uncertainty, In: Huynh, V.-N., Y. Nakamori, H. Ono, J. Lawry, V. Kreinovich, and H. T. Nguyen (eds.), Interval/Probabilistic Uncertainty and Non-Classical Logics, SpringerVerlag, Berlin-Heidelberg-New York, 2008, 32-56.

Pfanzangl, J. Theory of Measurement, John Wiley, New York, 1968.

Roth, A. Axiomatic Models of Bargaining, Springer-Verlag, Berlin, 1979.

Sawaragi, Y., H. Nakayama, and T. Tanino, Theory of Multiobjective Optimization, Academic Press, Orlando, Florida, 1985.

Steuer, R. E. Multiple Criteria Optimization: Theory, Computations, and Application, John Wiley \& Sons, New York, 1986.

Stevens, S. S. On the theory of scales of measurement. Science, 103:677-680, 1946. 
REC 2010 - A. Jaimes, C. Tweedie, T. Magoč, V. Kreinovich, and M. Ceberio 